\title{
EFEITO DE SISTEMAS DE PRODUÇÃO MISTOS SOB PLANTIO DIRETO SOBRE FERTILIDADE DO SOLO APÓS OITO ANOS ${ }^{(1)}$
}

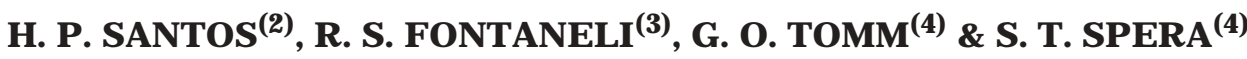

\begin{abstract}
RESUMO
Os atributos de solo relativos à sua fertilidade foram avaliados num Latossolo Vermel ho distrófico típico, em Passo Fundo (RS), oi to anos após o estabeleci mento (1993 a 2000) de cinco sistemas de produção que integravam culturas produtoras de grãos, pastagens de inverno e pastagens perenes - sistema I (trigo/soja, aveia branca/soja e ervilhaca/milho); sistema II (trigo/soja, aveia branca/soja e pastagem de aveia preta +ervilhaca/milho); sistema III [pastagens perenes da estação fria (festuca + trevo branco + trevo vermelho + cornichão)]; sistema IV [pastagens perenes da estação quente (pensacola + aveia preta + azevém + trevo branco + trevo vermelho + cornichão)]; e sistema V (alfafa para feno), que foi acrescentado como tratamento adicional, com repetições em áreas contíguas ao experimento, em 1994. As áreas sob os si stemas III, IV e V retornaram ao sistema I, a partir do verão de 1996. As culturas, tanto de inverno como de verão, foram estabelecidas sob plantio direto. Os tratamentos foram distribuídos em blocos ao acaso com quatro repetições. Os valores de $\mathrm{pH}$, de Al trocável, de $\mathrm{Ca}+\mathrm{Mg}$ trocáveis, de matéria orgânica, de $P$ extraível e de $K$ trocável foram influenciados pelos sistemas de produção. Os sistemas de produção elevaram os níveis de matéria orgânica, de P extraível e de K trocável, principalmente na profundidade de solo de 0-5 cm. Os teores de matéria orgânica e os de Al trocável, de P extraível e de K trocável diminuíram da camada de 0-5 $\mathrm{cm}$ para a camada de $15-20 \mathrm{~cm}$, enquanto para os valores de pH e de Ca + Mg trocáveis ocorreu o contrário.
\end{abstract}

Termos de indexação: rotação deculturas, integração lavoura-pecuária, pastagem anual, pastagem perene.

(1) Recebido para publicação em março de 2002 e aprovado em abril de 2003.

(2) Engenheiro-Agrônomo, Embrapa-Centro Nacional de Pesquisa de Trigo (Embrapa Trigo), Caixa Postal 451, CEP $99001-970$ Passo Fundo (RS). E.mail: hpsantos@cnpt.embrapa.br - Bolsista CNPq-PQ.

(3) Engenheiro-Agrônomo, Embrapa Trigo e Professor Titular da FAMV-UPF. E.mail: renatof@cnpt.embrapa.br

(4) Engenheiro-Agrônomo, Embrapa Trigo. E.mail: tomm@cnpt.embrapa.br; spera@cnpt.embrapa.br 


\title{
SUMMARY: EFFECT OF MIXED CROP PRODUCTION SYSTEMS UNDER NO-TILLAGE ON SOIL FERTILITY AFTER EIGHT YEARS
}

\begin{abstract}
Soil fertility characteristics were evaluated on a typical dystrophic Red Latosol (Typic Haplorthox) located in Passo F undo, State of Rio Grande do Sul, Brazil, after eight years of use under mixed production systems (1993 to 2000). The effects of production systems integrating grain production with winter annual and perennial pastures under no-tillage were assessed. Four production systems were evaluated: system I (wheat/ soybean, white oat/ soybean, and common vetch/ corn); system II (wheat/ soybean, white oat/ soybean, and grazed black oat + grazed common vetch/ corn); system III [perennial cool season pastures (fescue + white clover + red clover + birdsfoot trefoil)]; and system IV [perennial warm season pastures (bahiagrass + black oat + ryegrass + white clover + red cl over + birdsfoot trefoil)]; and system $V$ (alfalfa as hay crop), which was established in an adjacent area in 1994. Theareas under systems III I IV, and V returned to system I after thesummer of 1996. Thetreatments werearranged in a randomized completeblock design, with four replications. Exchangeable $\mathrm{Al}$ and $\mathrm{Ca}+\mathrm{Mg}$, organic matter, extractable $\mathrm{P}$, and exchangeable K leves wereinfluenced by crop production systems. Theproduction systems increased thecontents of organic matter, extractable $P$, and exchangeable $K$, mainly at $0-5 \mathrm{~cm}$ depth. Organic matter, exchangeable Al, extractable $\mathrm{P}$, and exchangeable $\mathrm{K}$ levels decreased from the 0 $5 \mathrm{~cm}$ layer to the $15-20 \mathrm{~cm}$ layer, while the opposite occurred with $\mathrm{pH}$ and exchangeable $\mathrm{Ca}+\mathrm{Mg}$ contents.
\end{abstract}

Index terms: crop rotation, ley farming, annual pastureand perennial pasture.

\section{INTRODUÇÃO}

No sistema plantio direto (PD), em virtude da localização dos fertilizantes adicionados, da concentração de resí duos vegetais e da menor erosão de solo, ocorre, na camada superficial, acúmulo de matéria orgânica e de cálcio + magnésio, de fósforo e de potássio (Sá, 1993; De Maria et al., 1999; Matowo et al., 1999; Santos \& Tomm, 1999; Silveira \& Stone, 2001). Além disso, a adição de fertilizantes nitrogenados amoniacais na superfície do solo promove frente de acidificação, com abaixamento do pH a partir da camada superficial, porém sem alterar a saturação por bases nem aumentar a toxidez por alumínio, provavelmente em decorrência da diminuição de sua atividade como resultado da presença de compostos orgânicos (Salet, 1994).

Nos trabal hos desenvolvidos por Sá (1993), com vários tipos de solo, no estado do Paraná, observouse aumento de 9 a 27 \% no teor de matéria orgânica no PD sobre o preparo convencional de solo. O acúmulo de matéria orgânica ocorreu na camada de 0-10 cm. De Maria et al. (1999), em Rhodic Ferralsol, em Campinas (SP), em PD, verificaram acúmulo de matéria orgânica, de $P$ e de $K$, na superfície do solo $(0-5 \mathrm{~cm})$, em relação à camada mais profunda (10$20 \mathrm{~cm}$ ). Santos \& Tomm (1999), no estado do Paraná, em Latossolo Bruno álico, em PD, observaram valores $\mathrm{Ca}+\mathrm{Mg}$ trocáveis diminuíram na camada de $0-5 \mathrm{~cm}$, em relação à camada de $15-20 \mathrm{~cm}$. Silveira \& Stone (2001), no estado de Goiás, em Latossolo Vermel ho perférrico, em PD, verificaram maiores teores de $\mathrm{Ca}+\mathrm{Mg}$, deP edeK na superfiície do solo $(0-10 \mathrm{~cm})$ do que na camada de $10-20 \mathrm{~cm}$.

Tem sido relatado o efeito de sistemas de rotação de culturas sobre características de fertilidade de sol o para produção degrãos; entretanto, são escassas as informações sobre esses efeitos em sistemas de produção mistos, ou seja, em que há integração de lavoura com pecuária. Alguns trabal hos relatam o efeito benéfico de pastagens perenes sobre a produtividade de algumas espécies (Carpenedo \& Mielniczuk, 1990), em razão da melhoria da fertilidade de sol o (Bayer \& Mielniczuck, 1997).

Este trabal ho teve como objetivo avaliar o efeito de sistemas de produção de grãos e de pastagens anuais de inverno e pastagens perenes, em sistema plantio direto (PD), após oito anos de cultivo, sobre características de fertilidade de solo e produtividade de culturas.

\section{MATERIAL E MÉTODOS}

O experimento foi realizado no Centro Nacional de Pesquisa de Trigo (E mbrapa Trigo), município de Passo F undo (RS) (longitude 28 o 15 ' S, latitude 52 24 ' W e altitude $684 \mathrm{~m}$ ), no período de 1993 a 2000, em Latossolo Vermelho distrófico típico (EMBRAPA, 1999), textura muito argilosa e relevo suave ondulado. Os teores médios de argila, silte e areia na camada de 0-20 cm são, respectivamente: 
720,130 e $150 \mathrm{~g} \mathrm{~kg}^{-1}$. As culturas componentes dos sistemas de produção que precederam o experimento foram: soja, no verão, e cevada ou trigo, no inverno.

Os tratamentos consistiram em quatro sistemas de produção que integravam grãos [aveia branca (Avena sativa L.), milho (Zea mays L.), soja (Glycine max Merrill) e trigo (Triticum aestivum L.)], pastagens anuais de inverno [aveia preta (Avena strigosa Schred.), azevém (Lolium multiflorum L.) e ervilhaca (Vicia sativa L.)] e pastagens perenes [alfafa (Medicago sativa L), cornichão (L otus corniculatus L.), festuca (F estuca arundinacea Schreb.), pensacola (Paspulum notatum Flügge), trevo branco (Trifolium repens L.) e trevo vermel ho (Trifolium pratense L.)]. São eles: sistema I (trigo/ soja, aveia branca/soja eervilhaca/milho); sistema II (trigo/soja, aveia branca/soja e pastagem de aveia preta + ervilhaca/milho); sistema III [pastagens perenes da estação fria (festuca + trevo branco + trevo vermel ho + cornichão)]; sistema IV [pastagens perenes da estação quente (pensacola + aveia preta + azevém + trevo branco + trevo vermelho + cornichão)]; e sistema V (alfafa para feno), acrescentado como tratamento adicional, com repetições em áreas contíguas ao experimento, em 1994 (Quadro 1). As áreas sob os sistemas III, IV e V retornaram aosistema I, a partir do verão de 1996. Todas as espécies, tanto no inverno como no verão, foram estabelecidas sob plantio direto.

Em abril de 1993, antes da semeadura das culturas de inverno, foram coletadas amostras de solo em cada parcela, à profundidade de $0-20 \mathrm{~cm}$, e os valores médios observados foram: $\mathrm{pH}=6,0$; Al trocável $=0,5 \mathrm{mmol}_{\mathrm{C}} \mathrm{dm}^{-3} ; \mathrm{Ca}+\mathrm{Mg}$ trocáveis = $102,8 \mathrm{mmol}_{\mathrm{c}} \mathrm{dm}^{-3}$; matéria orgânica $=23,0 \mathrm{~g} \mathrm{~kg}^{-1}$; P extraível $=5,3 \mathrm{mg} \mathrm{kg}^{-1}$; eK trocável $=60 \mathrm{mg} \mathrm{kg}^{-1}$. Três anos antes da instalação do experimento, foi efetuada calagem com cal cário dolomítico, baseada no método SMP (pH 6,0). As parcelas semeadas com al fafa foram corrigidas novamente com $6,0 \mathrm{t}$ ha-1 de calcário (PRNT $100 \%$ ), para elevar o pH para 6,5, aplicado em duas vezes: metade antes da aração (arado de discos) e metade antes da gradagem (grade niveladora).

A adubação de manutenção foi realizada de acordo com a recomendação para cada cultura (CFSRS/SC, 1995) e baseada nos resultados de análise de solo. As amostras de solo usadas para recomendação foram coletadas a cada três anos, depois da col heita das culturas de verão.

Em maio de 2000, após a col heita das culturas de verão, foram col etadas amostras de solo compostas de duas subamostras por parcela, em cada uma das seguintes profundidades: 0-5, 5-10, 10-15 e $15-20 \mathrm{~cm}$. As análises ( $\mathrm{pH}$ em água, P extraível, $\mathrm{K}$ trocável, matéria orgânica, Al trocável e Ca + Mg trocáveis) seguiram o método descrito por Tedesco et al. (1985): Al (extração em sol ução de KCl 1 mol L-1 e determinado por titulometria com solução $\mathrm{NaOH}$ 0,025 mol L-1); $\mathrm{Ca}+\mathrm{Mg}$ (mesmo extrator do Al e determinado por espectrofotometria de absorção atômica); matéria orgânica (determinada por combustão úmida); P eK (determinados por Mehlich-1) (Tedesco et al., 1985).

Quadro 1. Sistemas de produção de grãos e de pastagens anuais de inverno, perenes de estação fria e perenes de estação quente, sob plantio direto

\begin{tabular}{|c|c|c|c|c|c|c|c|c|}
\hline \multirow{2}{*}{ Sistema de produção } & \multicolumn{8}{|c|}{ Ano } \\
\hline & 1993 & 1994 & 1995 & 1996 & 1997 & 1998 & 1999 & 2000 \\
\hline $\begin{array}{l}\text { Sistema I } \\
\text { (produção de grãos) }\end{array}$ & $\begin{array}{l}\mathrm{T} / \mathrm{S} \\
\mathrm{E} / \mathrm{M} \\
\mathrm{Ab} / \mathrm{S}\end{array}$ & $\begin{array}{l}\mathrm{E} / \mathrm{M} \\
\mathrm{Ab} / \mathrm{S} \\
\mathrm{T} / \mathrm{S}\end{array}$ & $\begin{array}{l}\mathrm{Ab} / \mathrm{S} \\
\mathrm{T} / \mathrm{S} \\
\mathrm{E} / \mathrm{M}\end{array}$ & $\begin{array}{l}\mathrm{T} / \mathrm{S} \\
\mathrm{E} / \mathrm{M} \\
\mathrm{Ab} / \mathrm{S}\end{array}$ & $\begin{array}{l}\mathrm{E} / \mathrm{M} \\
\mathrm{Ab} / \mathrm{S} \\
\mathrm{T} / \mathrm{S}\end{array}$ & $\begin{array}{l}\mathrm{Ab} / \mathrm{S} \\
\mathrm{T} / \mathrm{S} \\
\mathrm{E} / \mathrm{M}\end{array}$ & $\begin{array}{l}\mathrm{T} / \mathrm{S} \\
\mathrm{E} / \mathrm{M} \\
\mathrm{Ab} / \mathrm{S}\end{array}$ & $\begin{array}{l}\mathrm{E} / \mathrm{M} \\
\mathrm{Ab} / \mathrm{S} \\
\mathrm{T} / \mathrm{S}\end{array}$ \\
\hline $\begin{array}{l}\text { Sistema II } \\
\text { (produção de grãos + } \\
\text { pastagem anual de inverno) }\end{array}$ & $\begin{array}{l}\mathrm{T} / \mathrm{S} \\
\mathrm{Ap}+\mathrm{E} / \mathrm{M} \\
\mathrm{Ab} / \mathrm{S}\end{array}$ & $\begin{array}{c}A p+E / M \\
A b / S \\
T / S\end{array}$ & $\begin{array}{c}\mathrm{Ab} / \mathrm{S} \\
\mathrm{T} / \mathrm{S} \\
\mathrm{Ap}+\mathrm{E} / \mathrm{M}\end{array}$ & $\begin{array}{c}\mathrm{T} / \mathrm{S} \\
\mathrm{Ap}+\mathrm{E} / \mathrm{M} \\
\mathrm{Ab} / \mathrm{S}\end{array}$ & $\begin{array}{l}\mathrm{Ap}+\mathrm{E} / \mathrm{M} \\
\mathrm{Ab} / \mathrm{S} \\
\mathrm{T} / \mathrm{S}\end{array}$ & $\begin{array}{c}\mathrm{Ab} / \mathrm{S} \\
\mathrm{T} / \mathrm{S} \\
\mathrm{Ap}+\mathrm{E} / \mathrm{M}\end{array}$ & $\begin{array}{l}T / S \\
A p+E / M \\
A b / S\end{array}$ & $\begin{array}{c}A p+E / M \\
A b / S \\
T / S\end{array}$ \\
\hline $\begin{array}{l}\text { Sistema III } \\
\text { (produção de grãos + } \\
\text { pastagem perene de inverno) }\end{array}$ & $\begin{array}{l}\text { T/PPF } \\
\text { T/PPF } \\
\text { T/PPF }\end{array}$ & $\begin{array}{l}\text { PPF } \\
\text { PPF } \\
\text { PPF }\end{array}$ & $\begin{array}{l}\text { PPF } \\
\text { PPF } \\
\text { PPF }\end{array}$ & $\begin{array}{l}\mathrm{PPF} / \mathrm{S} \\
\mathrm{PPF} / \mathrm{M} \\
\mathrm{PPF} / \mathrm{S}\end{array}$ & $\begin{array}{l}\mathrm{E} / \mathrm{M} \\
\mathrm{Ab} / \mathrm{S} \\
\mathrm{T} / \mathrm{S}\end{array}$ & $\begin{array}{l}\mathrm{Ab} / \mathrm{S} \\
\mathrm{T} / \mathrm{S} \\
\mathrm{E} / \mathrm{M}\end{array}$ & $\begin{array}{l}\mathrm{T} / \mathrm{S} \\
\mathrm{E} / \mathrm{M} \\
\mathrm{Ab} / \mathrm{S}\end{array}$ & $\begin{array}{l}\mathrm{E} / \mathrm{M} \\
\mathrm{Ab} / \mathrm{S} \\
\mathrm{T} / \mathrm{S}\end{array}$ \\
\hline $\begin{array}{l}\text { Sistema IV } \\
\text { (produção de grãos + } \\
\text { pastagem perene de verão) }\end{array}$ & $\begin{array}{l}\mathrm{T} / \mathrm{PPQ} \\
\mathrm{T} / \mathrm{PPQ} \\
\mathrm{T} / \mathrm{PPQ}\end{array}$ & $\begin{array}{l}P P Q \\
P P Q \\
P P Q\end{array}$ & $\begin{array}{l}P P Q \\
P P Q \\
P P Q\end{array}$ & $\begin{array}{l}\mathrm{PPQ} / \mathrm{S} \\
\mathrm{PPQ} / \mathrm{M} \\
\mathrm{PPQ} / \mathrm{S}\end{array}$ & $\begin{array}{l}\mathrm{E} / \mathrm{M} \\
\mathrm{Ab} / \mathrm{S} \\
\mathrm{T} / \mathrm{S}\end{array}$ & $\begin{array}{l}\mathrm{Ab} / \mathrm{S} \\
\mathrm{T} / \mathrm{S} \\
\mathrm{E} / \mathrm{M}\end{array}$ & $\begin{array}{l}\mathrm{T} / \mathrm{S} \\
\mathrm{E} / \mathrm{M} \\
\mathrm{Ab} / \mathrm{S}\end{array}$ & $\begin{array}{c}\mathrm{E} / \mathrm{M} \\
\mathrm{Ab} / \mathrm{S} \\
\mathrm{T} / \mathrm{S}\end{array}$ \\
\hline $\begin{array}{l}\text { Sistema V } \\
\text { (produção de grãos + alfafa) }\end{array}$ & $\begin{array}{l}- \\
- \\
-\end{array}$ & $\begin{array}{l}\mathrm{Al} \\
\mathrm{Al} \\
\mathrm{Al}\end{array}$ & $\begin{array}{l}\text { Al } \\
\text { Al } \\
\text { Al }\end{array}$ & $\begin{array}{l}\mathrm{Al} / \mathrm{S} \\
\mathrm{Al} / \mathrm{M} \\
\mathrm{Al} / \mathrm{S}\end{array}$ & $\begin{array}{l}\mathrm{E} / \mathrm{M} \\
\mathrm{Ab} / \mathrm{S} \\
\mathrm{T} / \mathrm{S}\end{array}$ & $\begin{array}{l}\mathrm{Ab} / \mathrm{S} \\
\mathrm{T} / \mathrm{S} \\
\mathrm{E} / \mathrm{M}\end{array}$ & $\begin{array}{l}\mathrm{T} / \mathrm{S} \\
\mathrm{E} / \mathrm{M} \\
\mathrm{Ab} / \mathrm{S}\end{array}$ & $\begin{array}{l}\mathrm{E} / \mathrm{M} \\
\mathrm{Ab} / \mathrm{S} \\
\mathrm{T} / \mathrm{S}\end{array}$ \\
\hline
\end{tabular}

Ab: aveia branca; Ap: aveia preta; Al: alfafa; E: ervilhaca; M: milho; PPF: pastagem de estação fria (festuca + cornichão + trevo branco); PPQ: pastagem estação quente (pensacola + cornichão + trevo vermelho); S: soja; e T: trigo. 
O del ineamento experimental adotado foi blocos ao acaso, com quatro repetições eparcelas de $400 \mathrm{~m}^{2}$. Os diversos sistemas de produção que integravam pastagens anuais de inverno e pastagens perenes com culturas produtoras de grãos foram comparados para cada característica de fertilidade de solo em determinada profundidade de amostragem. As profundidades de amostragem de solo foram comparadas em cada sistema de produção estudado. As médias foram comparadas entre si, pel o teste de Scheffé, a 5 \% (Gomes, 1973).

\section{RESULTADOS E DISCUSSÃO}

O valor médio de $\mathrm{pH}$ do solo (Quadro 2), para todas as camadas e sistemas de produção, apresentou valores menores do que o verificado nas camadas estudadas, após dois anos de cultivo, cujos valores foram de $0-5 \mathrm{~cm}$ : 5,96; $5-10 \mathrm{~cm}: 6,29 ; 10-$ $15 \mathrm{~cm} \mathrm{6,48;} \mathrm{e} \mathrm{15-20} \mathrm{cm:} \mathrm{6,29,} \mathrm{sob} \mathrm{plantio} \mathrm{direto}$ (Santos et al., 2001). Dentre os sistemas de produção estudados, houve diferença significativa para os valores depH dosolo somentena camada superficial. O tratamento $\mathrm{V}$ apresentou valor significativamente maior de $\mathrm{pH}$, na camada de $0-5 \mathrm{~cm}$ do que os sistemas III e IV. Nos quatro primeiros sistemas de produção, foi aplicado calcário há mais de oito anos e, no quinto sistema, em abril de 1994. Na avaliação de maio de 1998, o sistema V apresentou valor de $\mathrm{pH}$, na camada de $0-5 \mathrm{~cm}$, superior ao dos demais sistemas. I sso foi reflexo da aplicação de 6,0 t ha-1 de cal cário (PRNT $100 \%$ ), cerca de quatro anos antes.

Em todos os sistemas, houve perda gradual do efeito residual da calagem efetuada, em relação ao início do estabelecimento deste experimento e, principalmente, no sistema V. Em todos os sistemas, houveacidificação da camada de $0-5 \mathrm{~cm}$, necessitando aplicação de cal cário após sete anos para possi bilitar o cultivo eficiente delegumi nosas (CFSRS/SC, 1995). A acidificação do sol o nessa camada pode ter ocorrido em virtude da liberação de ácidos orgânicos por ocasião da decomposição da palhada das culturas antecedentes ou em decorrência do uso de fertilizantes nitrogenados (Paiva, et al., 1996; Franchini et al., 2000).

Em estudo realizado por Santos \& Tomm (1999), no estado do Paraná, sobre sistemas de rotação de culturas, incluindotrigo, sob PD, em Latossolo Bruno álico, no município de Guarapuava (PR), após cinco anos da aplicação de cal cário (11,7 t ha-1), verificouse que a quantidade recomendada não foi suficiente para aumentar o pH em nível desejado $(5,0$ a 6,0).

Em todos os sistemas de produção estudados, foram observadas diferenças significativas no val or de $\mathrm{pH}$ do solo entre determinadas profundidades de amostragem. A mesma tendência encontrada na avaliação de 1998 (Santos et al., 2001), em que os valores de $\mathrm{pH}$ aumentaram gradativamente com o aumento da profundidade do solo (0-5 e 10-15 cm), foi novamente observada. Dados semel hantes foram obtidos por Santos \& Tomm (1996) em sistemas de rotação de culturas incluindo trigo, sob sistema plantio direto (PD), em Latossolo Bruno álico, no município de Guarapuava (PR). Parte da resposta positiva das culturas à calagem pode ocorrer pelo aumento de absor ção de $\mathrm{N}$ pelas plantas (E dmeades et al., 1981). Além disso, a acidificação do sol o tende a reduzir a atividade microbiana para decomposição dos materiais orgânicos, liberação de nitrogênio mineral e absorção de N, que, por sua vez, limita o crescimento de plantas. Todavia, isso não foi verificado entre os sistemas de produção estudados, em razão do acúmulo de material orgânico na superfície como efeito do PD.

O valor de Al trocável do solo (Quadro 2), em todas as camadas de sol os amostrados e sistemas de produção estudados, sob PD, foi mais el evado do que na avaliação de maio de 1998, cujos valores foram de 0-5 cm: 0,54; 5-10 cm: 0,09; 10-15 cm: 0,00; e 15$20 \mathrm{~cm}: 0,14 \mathrm{mmol}_{\mathrm{c}} \mathrm{dm}^{-3}$ (Santos et al., 2001), nas quatro camadas estudadas. O aumento no teor de Al é conseqüência da acidificação. Com exceção da camada de $0-5 \mathrm{~cm}$ de profundidade, nas demais camadas não foram verificadas diferenças significativas nos teores de AI trocável do sol o entre os sistemas de produção. Na profundidade de 0 $5 \mathrm{~cm}$, o sistema III mostrou valor de Al trocável do sol o maior do que o do sistema $\mathrm{V}$.

Em dois dos cinco sistemas de produção, foram observadas diferenças significativas no teor de Al trocável entre as profundidades de amostragem do solo. Contudo, os sistemas I, II eV não diferiram entre as profundidades de amostragem. No sistema IV, o teor de AI trocável do solo diminuiu da camada de 0-5 cm para a camada de $15-20 \mathrm{~cm}$. Os sistemas IV e V favoreceram maior acidificação, pois há rel ação entreaumento do teor de Al trocável e diminuiução do pH. Santos \& Tomm (1996) obtiveram dados semelhantes somente para um sistema de rotação de culturas para trigo sob PD, em Latossolo Bruno álico, no município de Guarapuaca (PR).

Em trabalho realizado por Sidiras \& Pavan (1985), com sistemas de rotação deculturas incluindo trigo sob PD, em Latossolo Roxo distrófico, em Rolândia (PR), e Terra Roxa Estruturada, em Londrina (PR), os resultados foram inversos, ou seja, houve incremento no teor de Al trocável da camada de 0-10 para a de $10-20 \mathrm{~cm}$.

Nesta avaliação e em todos os sistemas de produção estudados, houve diminuição do valor de $\mathrm{pH}$ e aumento do teor de Al trocável na camada de 0-5 cm, em relação aos observados em maio de 1998, cujos valores foram de 5,95 e 0,54 $\mathrm{mmol}_{\mathrm{c}} \mathrm{dm}^{-3}$, 
Quadro 2. Valores médios de pH em água, de alumínio trocável, de cálcio + magnésio trocáveis, de matéria orgânica, de fósforo extraível e de potássio trocável avaliados após as culturas de verão de 2000, em quatro camadas de solo e para diferentes sistemas de produção

\begin{tabular}{|c|c|c|c|c|}
\hline \multirow{2}{*}{ Sistema de produção } & \multicolumn{4}{|c|}{ Profundidade $(\mathrm{cm})$} \\
\hline & $0-5$ & 5-10 & $10-15$ & $15-20$ \\
\hline & \multicolumn{4}{|c|}{$\mathrm{pH}$ - água, 1:1 } \\
\hline \multirow[t]{2}{*}{$\begin{array}{l}\text { I } \\
\text { II } \\
\text { III } \\
\text { IV } \\
\text { V }\end{array}$} & $\begin{array}{l}5,52 \mathrm{Cab} \\
5,54 \mathrm{Bab} \\
5,39 \mathrm{Bb} \\
5,43 \mathrm{Bb} \\
5,74 \mathrm{Ca}\end{array}$ & $\begin{array}{l}5,71 \mathrm{Bca} \\
5,89 \mathrm{ABa} \\
5,53 \mathrm{Ba} \\
5,63 \mathrm{Ba} \\
6,01 \mathrm{BCa}\end{array}$ & $\begin{array}{l}\text { 6,21 Aa } \\
6,18 \mathrm{Aa} \\
6,12 \mathrm{Aa} \\
6,13 \mathrm{Aa} \\
6,39 \mathrm{Aa}\end{array}$ & $\begin{array}{l}\text { 6,09 Aba } \\
6,03 \mathrm{Aa} \\
5,93 \mathrm{Aa} \\
6,28 \mathrm{Aa} \\
6,33 \mathrm{Aba}\end{array}$ \\
\hline & \multicolumn{4}{|c|}{ Alumínio, $\mathrm{mmol}_{\mathrm{c}} \mathrm{dm}^{-3}$} \\
\hline \multirow[t]{2}{*}{$\begin{array}{l}\text { I } \\
\text { II } \\
\text { III } \\
\text { IV } \\
\text { V }\end{array}$} & $\begin{array}{l}1,78 \mathrm{Aab} \\
1,51 \mathrm{Aab} \\
2,65 \mathrm{Aa} \\
2,43 \mathrm{Aab} \\
0,83 \mathrm{Ab}\end{array}$ & $\begin{array}{l}1,36 \mathrm{Aa} \\
0,83 \mathrm{Aa} \\
1,63 \mathrm{Aa} \\
1,99 \mathrm{ABa} \\
0,68 \mathrm{Aa}\end{array}$ & $\begin{array}{l}0,36 \mathrm{Aa} \\
0,40 \mathrm{Aa} \\
0,20 \mathrm{Ba} \\
0,40 \mathrm{Ba} \\
0,04 \mathrm{Aa}\end{array}$ & $\begin{array}{l}0,52 \mathrm{Aa} \\
0,92 \mathrm{Aa} \\
0,36 \mathrm{Ba} \\
0,33 \mathrm{Ba} \\
1,15 \mathrm{Aa}\end{array}$ \\
\hline & \multicolumn{4}{|c|}{ Cálcio + magnésio, $\mathrm{mmol}_{\mathrm{c}} \mathrm{dm}^{-3}$} \\
\hline \multirow[t]{2}{*}{$\begin{array}{l}\text { I } \\
\text { II } \\
\text { III } \\
\text { IV } \\
\text { V }\end{array}$} & $\begin{array}{l}62 \mathrm{Bb} \\
56 \mathrm{Bb} \\
62 \mathrm{Bb} \\
64 \mathrm{Bb} \\
79 \mathrm{Ba}\end{array}$ & $\begin{array}{l}70 \mathrm{ABb} \\
71 \mathrm{Ab} \\
70 \mathrm{ABb} \\
73 \mathrm{ABab} \\
88 \mathrm{ABa}\end{array}$ & $\begin{array}{l}78 \mathrm{Aab} \\
75 \mathrm{Ab} \\
80 \mathrm{Aab} \\
78 \mathrm{Aab} \\
95 \mathrm{Aa}\end{array}$ & $\begin{array}{l}77 \mathrm{Abb} \\
74 \mathrm{Ab} \\
76 \mathrm{Ab} \\
84 \mathrm{Aab} \\
94 \mathrm{Aa}\end{array}$ \\
\hline & \multicolumn{4}{|c|}{ Matéria orgânica, $\mathrm{g} \mathrm{kg}^{-1}$} \\
\hline \multirow[t]{2}{*}{$\begin{array}{l}\text { I } \\
\text { II } \\
\text { III } \\
\text { IV } \\
\text { V }\end{array}$} & $\begin{array}{l}33,6 \mathrm{Aa} \\
33,3 \mathrm{Aa} \\
36,7 \mathrm{Aa} \\
35,2 \mathrm{Aa} \\
34,5 \mathrm{Aa}\end{array}$ & $\begin{array}{l}28,8 \mathrm{Ba} \\
26,7 \mathrm{Ba} \\
30,2 \mathrm{Ba} \\
30,3 \mathrm{Ba} \\
29,6 \mathrm{Ba}\end{array}$ & $\begin{array}{l}25,4 \mathrm{BCa} \\
23,8 \mathrm{Ba} \\
24,9 \mathrm{Ca} \\
25,0 \mathrm{Ca} \\
26,7 \mathrm{Ca}\end{array}$ & $\begin{array}{l}23,9 \mathrm{Ca} \\
24,0 \mathrm{Ba} \\
24,8 \mathrm{Ca} \\
24,8 \mathrm{Ca} \\
26,6 \mathrm{Ca}\end{array}$ \\
\hline & \multicolumn{4}{|c|}{ Fósforo, mg kg-1 } \\
\hline $\begin{array}{l}\text { I } \\
\text { II } \\
\text { III } \\
\text { IV } \\
\text { V }\end{array}$ & $\begin{array}{l}18,4 \mathrm{Aab} \\
21,3 \mathrm{Aab} \\
15,1 \mathrm{Ab} \\
14,8 \mathrm{Ab} \\
24,6 \mathrm{Aa}\end{array}$ & $\begin{array}{r}12,3 \mathrm{Ba} \\
11,5 \mathrm{Ba} \\
8,8 \mathrm{Ba} \\
9,8 \mathrm{Ba} \\
13,0 \mathrm{Ba}\end{array}$ & $\begin{array}{l}\text { 5,7 Ca } \\
\text { 5,4 Ca } \\
\text { 4,9 BCa } \\
4,3 \mathrm{Ca} \\
4,5 \mathrm{Ca}\end{array}$ & $\begin{array}{l}3,5 \mathrm{Ca} \\
3,9 \mathrm{Ca} \\
3,3 \mathrm{Ca} \\
2,9 \mathrm{Ca} \\
3,2 \mathrm{Ca}\end{array}$ \\
\hline V & \multicolumn{4}{|c|}{ Potássio, mg kg-1 } \\
\hline $\begin{array}{l}\text { I } \\
I 1 \\
\text { III } \\
\text { IV } \\
\text { V }\end{array}$ & $\begin{array}{l}194 \mathrm{Ab} \\
174 \mathrm{Ab} \\
157 \mathrm{Ab} \\
158 \mathrm{Ab} \\
275 \mathrm{Aa}\end{array}$ & $\begin{array}{r}116 \mathrm{Bb} \\
109 \mathrm{Bb} \\
98 \mathrm{Bb} \\
86 \mathrm{Bb} \\
178 \mathrm{Ba}\end{array}$ & $\begin{array}{l}74 \mathrm{Cb} \\
74 \mathrm{BCb} \\
58 \mathrm{Cb} \\
53 \mathrm{BCb} \\
130 \mathrm{BCa}\end{array}$ & $\begin{array}{l}51 \mathrm{Cb} \\
55 \mathrm{Cab} \\
50 \mathrm{Cb} \\
42 \mathrm{Cb} \\
85 \mathrm{Ca}\end{array}$ \\
\hline
\end{tabular}

Médias seguidas da mesma letra maiúscula, na horizontal, e minúscula, na vertical, não apresentam diferenças significativas, a 5\%, pelo teste de Scheffé. I: trigo/soja, aveia branca/soja e ervilhaca/milho; II: trigo/soja, aveia branca/soja e pastagem de aveia preta + ervilhaca/milho; III: trigo/soja, aveia branca/soja e ervilhaca/milho após pastagem perene de inverno; IV: trigo/soja, aveia branca/ soja e ervilhaca/milho após pastagem perene de verão; e V: trigo/soja, aveia branca/soja e ervilhaca/milho após alfafa.

respectivamente(Santos et al., 2001), caracterizando acidificação nos primeir ros centímetros do solo. I sso pode ser atribuído à aplicação de fertilizantes nitrogenados, nos sistemas I ell , eà mineralização de resíduos culturais na superfície do solo (Salet, 1994). Para os demais sistemas de produção, essa explicação não é total mente válida, pois nesses não foram aplicados fertilizantes nitrogenados egrande parte da massa vegetal foi consumida pelos animais em pastejo.
Os teores de Ca + Mgtrocáveis do solo (Quadro 2), em todas as camadas, são considerados el evados para o crescimento e desenvol vimento de culturas tradicionais da região (CFSRS/SC, 1995). Contudo, esses teores estiveram abaixo do observado na camada de $0-5 \mathrm{~cm}$ dois anos antes, cujo teor foi $92 \mathrm{mmol}_{\mathrm{c}} \mathrm{dm}^{-3}$ (Santos et al., 2001). A acidez do solo da área experimental havia sido corrigida com calcário dolomítico oito anos antes do início do referido experimento. A aplicação de calcário 
dol omítico forneceu cálcio emagnési o em quantidades adequadas, de maneira que os teores ultrapassassem níveis críticos exigidos pelas espécies vegetais componentes dos sistemas de produção: 40 e $10 \mathrm{mmol}_{\mathrm{C}} \mathrm{dm}^{-3}$, respectivamente (CFSRS/SC, 1995).

Houve diferenças significativas entre os sistemas de produção quanto aos teores de Ca + Mg trocáveis do solo, em todas as profundidade de amostragem. Os sistemas I, II el II, porém, não diferiram entre si para os teores de $\mathrm{Ca}+\mathrm{Mg}$ trocáveis, nas camadas de $0-5,5-10$ e $15-20 \mathrm{~cm}$. O sistema $V$ apresentou teores maiores de $\mathrm{Ca}+\mathrm{Mg}$ trocáveis nas camadas de 0-5, 5-10 e 15-20 cm, em comparação aos sistemas I, II el II. I sso pode ser devido à aplicação de cal cário, em 1994, no sistema V. Resultados semelhantes foram obtidos por Silveira \& Stone (2001), em sistemas de rotação de culturas nos Cerrados de Goiás.

Comparando um mesmo sistema de produção, foram observadas diferenças significativas entre todas as profundidades de amostragem quanto aos teores de Ca + Mg trocáveis do solo. No sistema IV, os teores de $\mathrm{Ca}+\mathrm{Mg}$ trocáveis aumentaram da camada de 0-5 cm para $15-20 \mathrm{~cm}$. Esses dados estão de acordo com os obtidos por Santos \& Tomm (1996) em sistemas de rotação de culturas, incluindo trigo, após 4,5 anos, em Latotosso Bruno álico, no município de Guarapuava (PR). Todavia, Franchini et al. (2000), em sistemas de rotação incluindo soja, no Paraná, em Latossolo Roxo distrófico, nos municípios de L ondrina (PR), e Campo M ourão (PR), e Silveira \& Stone (2001), em sistemas de rotação de culturas com arroz e feijão, no Brasil Central, observaram maiores teores de Ca + Mgtrocáveis na camada de 0-10 cm, em relação aos da camada de 10-20 cm, em Latossolo Vermelho perférrico, no município de Santo Antônio de Goiás (GO). Por sua vez, os demais sistemas de produção estudados el evaram os teores de $\mathrm{Ca}+\mathrm{Mg}$ trocáveis até à camada de $10-15 \mathrm{~cm}$. Esses resultados foram o inverso dos obtidos para pH e para Al trocável, como previsto.

O teor de matéria orgânica do solo (Quadro 2), em todas as camadas e sistemas de produção, foi igual ou superior ao teor registrado dois anos antes, cujos valores foram: aos 0-5 cm: $32 \mathrm{~g} \mathrm{~kg}^{-1}$; aos 5-10 cm: $25 \mathrm{~g} \mathrm{~kg}^{-1}$; aos 10-15 cm: $23 \mathrm{~g} \mathrm{~kg}^{-1}$; e aos 15-20 cm: $24 \mathrm{~g} \mathrm{~kg}^{-1}$ (Santos et al., 2001). Na maioria dos estudos sob PD, tem sido observado acúmulo de matéria orgânica nas camadas próximas à superfície do solo. Por sua vez, esse acúmulo de matéria orgânica no sistema plantio direto aumenta a força iônica da solução de solo na camada superficial (Salet, 1994). I sso explica, em parte, a nãoocorrência de toxidez de alumínio, em razão da menor atividade iônica do alumínio.

Nos sistemas de produção estudados, em todas as camadas de amostragem, não houve diferenças significativas entre os valores médios de matéria orgânica do solo. Santos et al. (1999), trabal hando com sistemas de rotação de cul turas, incluindo trigo, observaram dados semelhantes para o teor de matéria orgânica, em todas as camada estudadas.

Foram verificadas diferenças significativas no teor de matéria orgânica entre determinadas profundidades de amostragem do solo em todos os sistemas de produção estudados. Para o teor de matéria orgânica, na maioria dos sistemas, houve redução progressiva da camada superficial para a camada mais profunda. Resultado semel hante nas variações entreteor de matéria orgânica da camada de $0-5 \mathrm{~cm}$ para a camada de $15-20 \mathrm{~cm}$ foi verificado por Sá (1993) e por Santos \& Tomm (1996), em sistemas de rotação de culturas para trigo. A manutenção do teor de matéria orgânica em valores mais el evados apenas na camada superficial do solo decorre do acúmulo de resíduos vegetais sobre a superfície do sol o sob PD, resultante da ausência de incorporação destes por meio do revolvimento, que é praticado no preparo convencional de solo, a qual diminui a taxa de mineralização. Resultados similares foram obtidos por Bayer \& Mielniczuk (1997).

O teor deP extraível dosolo na camada superficial (0-5 cm), em todos os sistemas estudados, foi superior ao valor considerado crítico $\left(9,0 \mathrm{mg} \mathrm{kg}^{-1}\right)$ nesse tipo de solo para crescimento e desenvolvimento de culturas tradicionais (CFSRS/SC, 1995) (Quadro 2). O teor deP extraível do solo na maioria dos sistemas ( I II e V) aumentou, nas camadas de 0-5 e 5-10 cm, em relação ao teor medido em 1998, cujos valores atingiram 15,3; 8,1; 5,3 e4, $2 \mathrm{mg} \mathrm{kg}^{-1}$, respectivamente (Santos et al., 2001). Como tem sido observado, o PD provoca alterações nas propriedades químicas do solo, as quais, por sua vez, refletem-se na fertilidade e na eficiência de uso de nutrientes pelas espécies (Eckert, 1991; Sá, 1993; Paiva et al., 1996). A rotação de culturas tem importante papel na ciclagem de nutrientes, uma vez que as espécies vegetais diferem entre si no que se refere à quantidade eà qualidade de resíduos fornecidos, à eficiência de absorção de íons e à exploração de diferentes profundidades de sol o pel o sistema radicular.

Houve diferenças significativas entre os sistemas de produção estudados para o valor do $\mathrm{P}$ extraível do solo, apenas na camada de $0-5 \mathrm{~cm}$. O teor de $P$ extraível do solo, nessa camada, foi maior no sistema $V$ do quenos sistemas III eIV. De Maria et al. (1999), trabal hando com sistemas de manejo de solo e de rotação de culturas, encontraram menor teor de $\mathrm{P}$ extraível na monocultura de milho do que em milho após soja, sob PD, em Rhodic Ferralsol, em Campinas (SP).

Os sistemas de produção avaliados diferiram significativamente quanto ao teor de $P$ extraível em todas as profundidades de amostragem (I: 18,4 a $3,5 \mathrm{mg} \mathrm{kg}^{-1}$; II: 21,3 a 3,9 $\mathrm{mg} \mathrm{kg}^{-1}$; III: 15,1 a $3,3 \mathrm{mg} \mathrm{kg}^{-1}$; IV: 14,8 a 2,9 $\mathrm{mg} \mathrm{kg}^{-1}$; e V: 24,6 a 
$\left.3,2 \mathrm{mg} \mathrm{kg}^{-1}\right)$. Em todos os sistemas, o valor de P extraível na camada de $0-5 \mathrm{~cm}$ foi 5,1 a 7,7 vezes maior do que o teor registrado na camada de 15$20 \mathrm{~cm}$. Resultados semel hantes foram obtidos em outros estudos em PD por Sá (1993), por Matowo et al. (1999) e por Santos \& Tomm (1999). Segundo Sidiras \& Pavan (1985), o acúmulo de P extraível próximo à superfície do solo decorre das aplicações anuais de fertilizantes fosfatados, da liberação de $P$ durante a decomposição de resíduos vegetais e da menor fixação deP, em razão do menor contato desse elemento com os constituintes inorgânicos do solo, uma vez que não há incorporação de resíduos vegetais por meio do revolvimento de solo no PD (Wisniewski \& Holtz, 1997).

O teor deK trocável do sol o observado na camada de $0-5$ e $5-10 \mathrm{~cm}$ (Quadro 2), em todos os sistemas estudados, foi superior ao considerado crítico $\left(80 \mathrm{mg} \mathrm{kg}^{-1}\right)$ para crescimento e desenvol vimento de culturas (CF SRS/SC, 1995). Além disso, oteor deK trocável observado em todos os sistemas de produção e nas camadas de 0-5 a 10-15 cm manteve-se acima do encontrado na avaliação de 1998, cujos valores foram de 106, 65 e $47 \mathrm{mg} \mathrm{kg}^{-1}$, respectivamente (Santos et al., 2001).

Neste período de estudo, os teores de K trocável do solo diferiram significativamente entre alguns sistemas produção. O teor de K trocável, nas camadas de $0-5$ a $10-15 \mathrm{~cm}$, foi mais el evado no sistema V. Essas diferenças em favor da cultura de alfafa podem estar relacionadas com o maior teor de K trocável propiciado pela adubação de manutenção ou pel o resíduo cultural dessa leguminosa. Silveira $\&$ Stone (2001) encontraram diferenças significativas para K trocável entre sistemas de rotação de culturas, somente na camada de $10-20 \mathrm{~cm}$, em Latossolo Vermelho perférrico, no município de Santo Antônio de Goiás (GO).

Foram verificadas diferenças significativas em teor de K trocável entre todas as profundidades de amostragem desol o detodos os sistemas de produção avaliados. A exemplo do verificado com P extraível, também houve acúmulo de $K$ trocável na camada próxima à superfície nos diferentes sistemas avaliados. O teor de $\mathrm{K}$ trocável, na camada de $0-5 \mathrm{~cm}$, foi 3,2 vezes maior que o verificado na camada de $15-20 \mathrm{~cm}$. Acúmulo semel hante de $\mathrm{K}$ trocável, na camada de $0-5 \mathrm{~cm}$, em relação à camada de $15-20 \mathrm{~cm}$, em sistemas de rotação de culturas sob PD, foi observado por De Maria et al. (1999) e por Santos \& Tomm (1999).

\section{CONCLUSÕES}

1. Os valores de $\mathrm{pH}$, teores de Al trocável, de $\mathrm{Ca}+\mathrm{Mg}$ trocáveis, de matéria orgânica, de $\mathrm{P}$ extraível edeK trocável foram influenciados pel os sistemas de produção.
2. Os teores de matéria orgânica do solo, de $P$ extraível e de $K$ trocável, principalmente na camada de solo de 0-5 cm, aumentaram em relação aos teores observados em dois anos, de 1998 a 2000, em todos os sistemas de produção realizados sob sistema plantio direto.

3. Os teores de matéria orgânica do solo, de Al trocável, deP extraível edeK trocável diminuíram progressivamente da camada de 0-5 para a de 15-20 cm, enquanto, para os valores de $\mathrm{pH}$ e teores de Ca + Mg trocáveis, ocorreu o inverso, em dois anos.

\section{LITERATURA CITADA}

BAYER, C. \& MIELNICZUK, J. Nitrogênio total de um solo submetido a diferentes métodos de preparo e sistemas de cultura. R. Bras. Ci. Solo, 21:235-239, 1997.

CARPENEDO, V. \& MIELNICZUK, J. Estado de agregação e qualidade de agregados de Latossolos Roxos submetidos a diferentes sistemas de manejo. R. Bras. Ci. Solo, 14:99105, 1990.

COMISSÃO DE FERTILIDADE DO SOLO - CFSRR/SC. Recomendações de adubação e de calagem para os estados do Rio Grande do Sul e de Santa Catarina. 3.ed. Passo Fundo, Sociedade Brasileira de Ciência do solo - Núcleo Regional Sul, 1995. 224p.

DE MARIA, I.C.; NNABUDE, P.C. \& CASTRO, O.M. Long-term tillage and crop rotation effects on soil chemical properties of a Rhodic Ferralsol in southern Brazil. Soil Till. Res., 51:71-79, 1999.

ECKERT, D.J . Chemical attributes of soils subjected to no-till cropping with rye cover crops. Soil Sci. Soc. Am. J ., 55:405409, 1991.

EDMEADES, D.C.; J UDO, M. \& SARATHCHANDRA, S.U. The effect of lime on nitrogen mineralization as measured by grass growth. Plant Soil, 60:177-186, 1981.

EMPRESA BRASILEIRA DE PESQUISA AGROPECUÁRIA EMBRAPA. CentroNacional de Pesquisa de Solos. Sistema brasileiro de classificação de solos. Brasília, Embrapa Produção da Informação, Rio de J aneiro, 1999. 412p.

FRANCHINI, J.C.; BORKERT, C.M.; FERREIRA, M.M. \& GAUDÊNCIO, C.A. Alterações na fertilidade do solo em sistemas de rotação de culturas em semeadura direta. $\mathrm{R}$. Bras. Ci. Solo, 24:459-467, 2000.

GOMES, F.P. Curso de estatística experimental. 5.ed. Piracicaba, USP-ESALQ, 1973. 430p

MATOWO, P.R.; PIERZYNSKI, G.M.; WHITNEY, D. \& LAMOND, R.E. Soil chemical properties as influenced by tillage and nitrogen source, placement, and rates after 10 years of continuous sorghum. Soil Till. Res., 50:11-19, 1999.

PAIVA, P.J .R.; VALE, F.R.; FURTINI NETO, A.E. \& FAQUIN, V. Acidificação de um Latossolo Roxo do estado do Paraná sob diferentes sistemas de manejo. R. Bras. Ci. Solo, 20:7175, 1996. 
SÁ, J .C.M. Manejo da fertilidade do solo no sistema plantio direto. In: EMPRESA BRASILEIRA DE PESQUISA AGROPECUÁRIA - EMBRAPA. Centro Nacional de Pesquisa de Trigo. Plantio direto no Brasil. Passo Fundo, 1993. p.37-60.

SALET, R.L Dinâmica de íons na solução de um sol o submetido ao sistema plantio direto. Porto Alegre, Universidade Federal do Rio Grande Sul, 1994. 111p. (Tese de Mestrado)

SANTOS, H.P.; FONTANELI, R.S. \& TOMM, G.O. Efeito de sistemas de produção de grãos e de pastagens sob plantio direto sobre o nível de fertilidade do solo após cinco anos. R. Bras. Ci. Solo, 25:645-653, 2001.

SANTOS, H.P. \& TOMM, G.O. Estudos da fertilidade do solo sob quatro sistemas de rotação de culturas envol vendo trigo em plantio direto. R. Bras. Ci. Solo, 20:407-414, 1996.
SANTOS, H.P.\& TOMM, G.O. Rotação de culturas para trigo, após quatro anos: efeitos na fertilidade do solo em plantio direto. Ci. Rural, 29:259-265, 1999.

SIDIRAS, N. \& PAVAN, M.A. I nfluência do sistema de manejo do sol o no seu nível de fertilidade. R. Bras. Ci. Solo, 9:249254, 1985.

SILVEIRA, P.M. \& STONE, L.F. Teores de nutrientes e de matéria orgânica afetados pela rotação deculturas esistemas de preparo do solo. R. Bras. Ci. Solo, 25:387-394, 2001.

TEDESCO, M.J .; VOLKWEISS, S.J . \& BOHNEN, H. Análise de solos, plantas e outros materiais. Porto Alegre, Universidade Federal do Rio Grande do Sul, 1985. 32p. (Boletim Técnico, 5)

WISNIEWSKI, C. \& HOLTZ, G.P. Decomposição da palhada com liberação de nitrogênio e fósforo numa rotação aveia-soja sob plantio direto. Pesq. Agropec. Bras., 32:1191-1197, 1997. 